\title{
Miliary Tuberculosis-Related Acute Respiratory Distress Syndrome: Early Diagnosis Can Save Life
}

\author{
Divya Sethi, Shivi Goyal \\ Department of Anesthesia and Critical Care, Employees' State Insurance Postgraduate Institute of Medical Sciences and Research, New Delhi, India
}

\section{Abstract}

Miliary tuberculosis (TB) is an uncommon cause of acute respiratory distress syndrome (ARDS) with a high mortality. Early diagnosis and timely initiation of treatment are important for good outcome. We report the case of a 23 -year-old female who needed admission to intensive care unit (ICU) due to ARDS. On routine investigations, the cause of ARDS could not be ascertained. Finally, high-resolution computed tomography of the chest and bronchoscopic-guided lung biopsy were done which confirmed the etiology to be miliary TB. The patient showed an improvement after starting antitubercular therapy with steroids. One week later, the patient's condition was stabilized and was shifted out of ICU. This case report emphasizes that miliary TB, though difficult to diagnose, should always be considered a differential diagnosis in patients with ARDS, as early initiation of treatment can prove to be lifesaving.

Keywords: Acute respiratory distress syndrome, antitubercular drug, intensive care unit, miliary tuberculosis

\section{INTRODUCTION}

Massive lymphohematogenous dissemination of Mycobacterium tuberculosis bacilli leads to miliary tuberculosis (TB). It is a potentially lethal disease if not diagnosed and treated early. It accounts for $<2 \%$ of all TB cases and $20 \%$ of all extrapulmonary TB cases in immune-competent adults; the infection rate in immunocompromised patients is much higher. ${ }^{[1]}$ Miliary TB can sometimes lead to acute respiratory distress syndrome (ARDS). ${ }^{[2]}$ Patients of miliary TB with ARDS have a high mortality of $33 \%-90 \%{ }^{[3]}$ We present a case of miliary TB with ARDS and discuss its diagnosis and management.

\section{Case Report}

A 23-year-old female at 8 weeks of gestation was diagnosed with hydatidiform mole, for which she underwent suction and evacuation of the uterus. After an uneventful postoperative period, she was discharged from the hospital but returned back after 4 weeks with complaints of high-grade fever and dry cough. For investigating the possible source of infection, cultures of blood, sputum, urine, and high vaginal swab were sent, and broad-spectrum antibiotics were started. The patient's hematological investigations were normal except for low hemoglobin of $7.7 \mathrm{gm} \%$, for which one unit of packed red

\begin{tabular}{|l|l|}
\hline \multicolumn{2}{|c|}{ Access this article online } \\
\hline Quick Response Code: & Website: \\
& www.ijrc.in \\
& \\
\hline
\end{tabular}

cells was transfused. Her chest X-ray was unremarkable and culture reports were negative, but fever persisted in spite of antibiotic therapy.

Two weeks later, the patient developed tachypnea and her chest $\mathrm{X}$-ray showed fluffy infiltrates over bilateral lung fields. She was transferred to intensive care unit (ICU), and noninvasive mask ventilation with pressure support of $15 \mathrm{~cm}$ of $\mathrm{H}_{2} \mathrm{O}$ and $\mathrm{FiO}_{2}$ of 0.6 was started. Her arterial blood gas analysis was indicative of ARDS $\left(\mathrm{PaO}_{2}\right.$ of $55 \mathrm{mmHg}$ at $\mathrm{FiO}_{2}$ of $\left.0.6 ; \mathrm{PaO}_{2} / \mathrm{FiO}_{2}=92.5\right)$. Repeat cultures (blood, sputum, and urine) were sent which were again negative for any bacteriological growth. Other tests including Widal, malaria antigen, and dengue serology were also negative. In spite of broad-spectrum antibiotic therapy, her condition did not improve. On day 5 of ICU admission, a high-resolution computed tomography (HRCT) scan of the

Address for correspondence: Dr. Divya Sethi, Department of Anesthesia and Critical Care, Employees' State Insurance Postgraduate Institute of Medical Sciences and Research, New Delhi - 110 015, India. E-mail: divyasth@gmail.com

This is an open access journal, and articles are distributed under the terms of the Creative Commons Attribution-NonCommercial-ShareAlike 4.0 License, which allows others to remix, tweak, and build upon the work non-commercially, as long as appropriate credit is given and the new creations are licensed under the identical terms.

For reprints contact: WKHLRPMedknow_reprints@wolterskluwer.com

How to cite this article: Sethi D, Goyal S. Miliary tuberculosis-related acute respiratory distress syndrome: Early diagnosis can save life. Indian J Respir Care 2021;10:243-5.

Received: $13-05-2020 \quad$ Revised: $29-09-2020$ Accepted: $30-10-2020 \quad$ Published: $14-06-2021$ 
chest was done which showed diffuse ground-glass opacity with septal thickening in bilateral lung parenchyma along with basilar consolidation and miliary nodules pathognomic of miliary TB [Figures 1 and 2]. A bronchoscopy-guided lung tissue sample was taken and sent for microscopic examination which showed epithelial cell granuloma with caseating necrosis confirming TB [Figure 3]. The bronchoalveolar lavage sample tested positive polymerase chain reaction (PCR)-TB; however, sputum for acid-fast bacilli was negative. Antitubercular drugs and steroids were started; thereafter, there was an improvement in the patient's symptoms with resolution of fever and breathlessness. After 1 week of initiating antitubercular therapy, the patient was transferred to ward on room air oxygen and later discharged with an advice to continue antitubercular therapy.

\section{Discussion}

ARDS is a life-threatening reaction to injuries or infections of the lung with a high mortality rate of $30 \%-40 \%$. Miliary TB is being increasingly recognized as a cause of ARDS in india.

The pathogenesis of ARDS in miliary TB is not completely understood. The suggested pathways include massive release of Mycobacterium into circulation leading to inflammation, obliterative endarteritis, and alveolocapillary membrane damage. In addition, lipoarabinomannan component of Mycobacterium cell wall triggers increased macrophage activity and massive release of cytokines including tumor necrosis factor- $\alpha$ and interleukin- $1 \beta{ }^{[3]}$ Finally, the pathogen burden, virulence of the Mycobacterium strain, and the immunologic responses mounted by infected individuals determine the development and progress of lung injury.

The criteria used for diagnosing miliary TB include the presence of clinical symptoms of TB, classical miliary pattern, or bilateral diffuse reticulonodular lesions on a background of miliary shadows on plain chest X-ray or HRCT scan; histopathological or microbiological evidence of TB; and favorable clinical or radiographic response to antitubercular treatment. ${ }^{[4]}$ Although military TB can cause ARDS, its diagnosis may be challenging and often missed by clinicians due to atypical clinical presentation of the disease or the absence of classical miliary pattern on chest X-ray. The differentiating clinical features between miliary TB and bacterial pneumonia include the longer duration of constitutional symptoms such as fever with malaise, cough with breathlessness, and weight loss and the absence of response to empirical antibiotic therapy. ${ }^{[5,6]}$

The classical miliary pattern on chest radiograph is present in only $50 \%$ of patients with miliary TB and may be apparent only weeks after tubercular infection. ${ }^{[7]}$ Furthermore, the miliary pattern on chest X-ray may get masked once ARDS has developed. In these situations, HRCT is the useful radiological investigation for the diagnosis of active disease. Although mycobacterial culture is the gold standard for the diagnosis of TB, this cannot be relied upon for initiating the treatment in ARDS with miliary TB as culture results take as

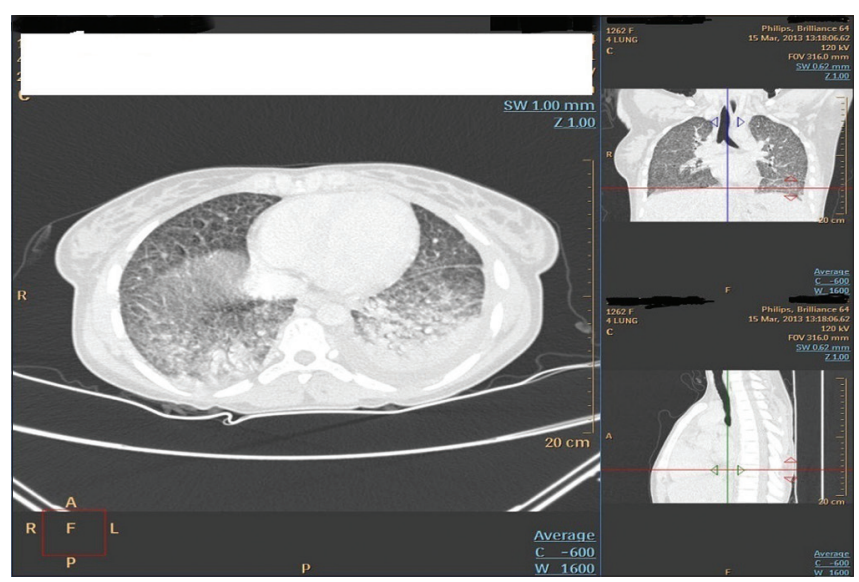

Figure 1: High-resolution computed tomography showing the transverse section of the lung with bilateral military nodules and basal consolidations

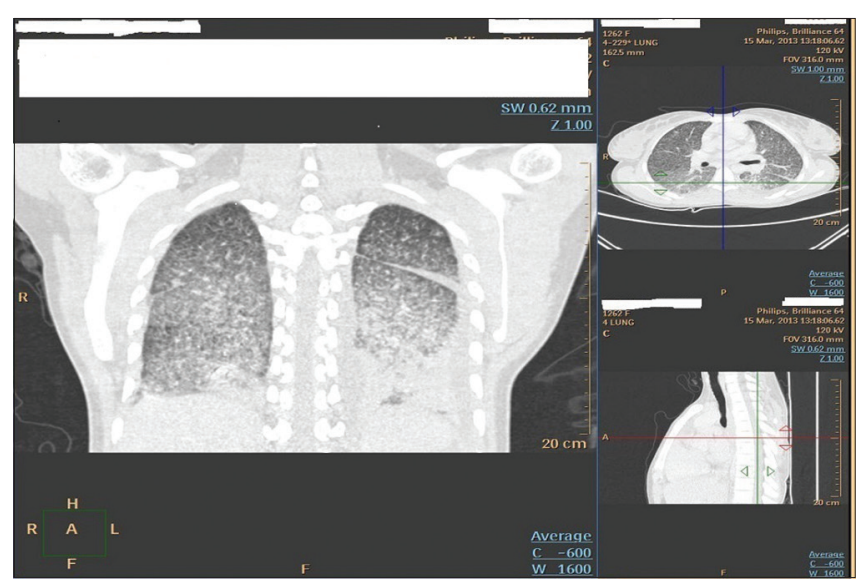

Figure 2: High-resolution computed tomography showing the coronal section of the lung with septal thickening and miliary nodules

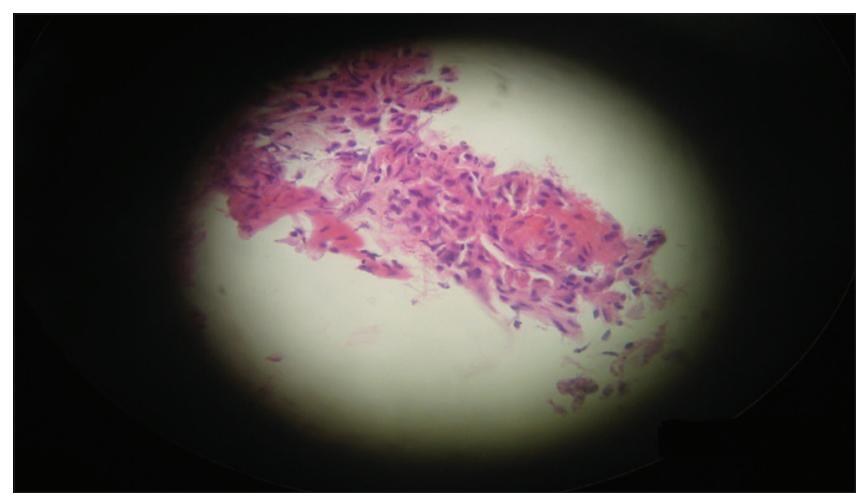

Figure 3: Histopathology slide of lung tissue showing epithelial cell granuloma with necrosis

long as $6-8$ weeks. In our patient, the diagnosis of miliary TB was made on the basis of findings of HRCT and microscopic pathological examination of bronchoscopic tissue sample which showed caseating granulomas [Figure 3].

Treatment with antitubercular drugs is an important factor that can affect patient outcome. Higher mortality within 1 year 
is found among patients who do not receive appropriate antitubercular treatment. ${ }^{[8]}$ In patients with ARDS with suspicion of miliary TB as the ethology, antitubercular treatment should be started empirically, even before the availability of confirmatory test results, as it increases the survival likelihood. ${ }^{[9]}$ In the treatment of military TB with ARDS, therapy with corticosteroid is shown to be beneficial. Corticosteroids act by inhibiting the release of lymphokines and cytokines, which are responsible for constitutional symptoms and tissue damage. In addition, corticosteroids allow the penetration of antitubercular drugs into the granulomas and its disruption. ${ }^{[5]}$ Administration of corticosteroids in early stages has shown to improve pulmonary and extrapulmonary organ dysfunction in patients with ARDS. ${ }^{[10]}$

\section{Conclusion}

Prognosis of miliary TB with ARDS can be improved by maintaining high index of suspicion of TB in cases of acute respiratory failure of unknown origin, particularly in immunocompromised individuals. In addition, HRCT and bronchoscopic-guided lung biopsy are useful modalities in difficult to diagnose cases. Early initiation of treatment with antitubercular drugs can improve the outcome.

\section{Declaration of patient consent}

The authors certify that they have obtained all appropriate patient consent forms. In the form the patient(s) has/have given his/her/their consent for his/her/their images and other clinical information to be reported in the journal. The patients understand that their names and initials will not be published and due efforts will be made to conceal their identity, but anonymity cannot be guaranteed.

\section{Financial support and sponsorship} Nil.

\section{Conflicts of interest}

There are no conflicts of interest.

\section{References}

1. Sharma SK, Mohan A, Sharma A. Challenges in the diagnosis treatment of miliary tuberculosis. Indian J Med Res 2012;135:703-30.

2. Kim JY, Park YB, Kim YS, Kang SB, Shin JW, Park IW, et al. Miliary tuberculosis and acute respiratory distress syndrome. Int J Tuberc Lung Dis 2003;7:359-64.

3. Zang YM, Doerfler TC, Leu B, Guillenm B, Ron WN. Mechanism of stimulation of interleukin and 1 | 3 and TNF-a by Mycobacterium tuberculosis components. J Clin Invest 1993;91:2076.

4. Sharma SK, Mohan A, Pande JN, Prasad KL, Gupta AK, Khilnani GC. Clinical profile, laboratory characteristics and outcome in miliary tuberculosis. QJM 1995;88:29-37.

5. Maartens G, Willcox PA, Benatar SR. Miliary tuberculosis: Rapid diagnosis, hematologic abnormalities, and outcome in 109 treated adults. Am J Med 1990;89:291-6.

6. Roger PM, Deloffre P, Tiger F, Bernardin G, Corcelle P, Dellamonica P, et al. Prognosis of acute tuberculous respiratory distress syndrome. 4 cases. Presse Med 1995;24:1021-4.

7. Kim JH, Langston AA, Gallis HA. Miliary tuberculosis: Epidemiology, clinical manifestations, diagnosis, and outcome. Rev Infect Dis 1990;12:583-90.

8. Sharma SK, Mohan A. Miliary tuberculosis. In: Schlossberg D, editor. Tuberculosis and Nontuberculous Mycobacterial Infections. $6^{\text {th }}$ ed.. Washington: American Society for Microbiology Press; 2011. p. 415-35.

9. Blumberg HM, Burman WJ, Chaisson RE, Daley CL, Etkind SC, Friedman LN, et al. American Thoracic Society/Centers for Disease Control and Prevention/Infectious Diseases Society of America: Treatment of tuberculosis. Am J Respir Crit Care Med 2003;167:603-62.

10. Meduri GU, Golden E, Freire AX, Taylor E, Zaman M, Carson SJ, et al. Methylprednisolone infusion in early severe ARDS: Results of a randomized controlled trial. Chest 2007;131:954-63. 